\title{
Responsabilidad social en la gestión ambiental Atavillos Bajo, Lima,2021
}

\author{
Saara Huaches LLocya \\ shuachesll@ucvvirtual edu.pe \\ Deisy Neydina Tolentino Luna \\ dntolentinot@ucvvirtual.edu.pe \\ Walter Manuel Vásquez Mondragón \\ wvasquezmo@ucvvirtual.edu.pe \\ Lidia Amanda Díaz Saavedra \\ idiazsaa@ucvvirtual.edu.pe \\ Nelberto Ortiz Zavaleta \\ nortizz@ucvvirtual.edu.pe \\ Universidad Cesar Vallejo \\ Lima- Perú
}

\section{RESUMEN}

La presente investigación tuvo como objetivo genérico demostrar cómo la responsabilidad social incide en la gestión ambiental teniendo objetivos específicos para ser redactados en base mediante el estudio de la responsabilidad social y su incidencia en la gestión ambiental. Atavillos Bajo, Lima, 2021. Se presentó con un paradigma positivista, diseño no experimental, con enfoque cuantitativo y es explicativo correlacional-causal. La población total fue conformada por 84 pobladores. Los instrumentos utilizados se validaron por jueces (tipo cualitativa) y se dio sustento a través de prueba binomial (contenido de tipo cuantitativo), cuyo valor fue menor a 0,05 confirmándose que el instrumento fue válido.

Los principales resultados muestran que el $50 \%$ de los pobladores del distrito, manifiestan que la responsabilidad social, es deficiente, asimismo. Mientras que el $47,62 \%$ refiere que el nivel es moderado. También, el 2,38\% menciona que es muy eficiente. Así mismo, ante la prevalencia de los datos presentados en tabla y figura podemos manifestar que la responsabilidad social, es deficiente en la gestión ambiental. Se concluyó La responsabilidad social incide de manera significativa (p-valor < 0.05), 
Huaches LLocya y otros...

positiva con la gestión ambiental en el distrito; con lo cual la hipótesis general queda demostrada

Palabras clave: responsabilidad; cultura ambiental; desarrollo sostenible; gestión ambiental. 


\title{
Social responsibility in environmental management.
}

Atavillos Bajo, Lima, 2021

\begin{abstract}
The present research had the generic objective of demonstrating how social responsibility affects environmental management, having specific objectives to be drafted based on the study of social responsibility and its impact on environmental management. Atavillos Bajo, Lima, 2021. It was presented with a positivist paradigm, non-experimental design, with quantitative approach and is explanatory correlationalcausal. The total population consisted of 84 inhabitants. The instruments used were validated by judges (qualitative type) and were supported by a binomial test (quantitative type content), whose value was less than 0.05 , confirming that the instrument was valid.

The main results show that $50 \%$ of the district's inhabitants state that social responsibility is deficient, while $47.62 \%$ say that social responsibility is deficient. Meanwhile, $47.62 \%$ say that the level is moderate. Also, $2.38 \%$ mentioned that it is very efficient. Likewise, given the prevalence of the data presented in the table and figure, we can state that social responsibility is deficient in environmental management. It was concluded that social responsibility has a significant ( $\mathrm{p}$-value $<0.05$ ), positive impact on environmental management in the district; with which the general hypothesis is demonstrated.
\end{abstract}

Keywords: responsibility, environmental culture, sustainable development, environmental management

Artículo recibido: 02 enero 2022 Aceptado para publicación: 28 enero 2022 Correspondencia: shuachesll@ucvvirtual edu.pe Conflictos de Interés: Ninguna que declarar 
Huaches LLocya y otros...

\section{INTRODUCCIÓN}

En el contexto actual, la responsabilidad social es un tema importante y esto se refleja en el compromiso que tienen las sociedades para dar soluciones a los diversos flagelos presentados en determinados grupos de interés. Siendo la contaminación un problema muy complejo que viene generando daño, cuyos inicios son considerados en la Primera Revolución Industrial donde se originó más emisiones y poco a poco se ha ido incrementado (Canales, 2021; Liu et al., 2018; Sala, 2020).

Por otro lado, el desarrollo de industrias ha generado la migración a diferentes espacios geográficos degradándose aún más el ambiente para construir viviendas, con el pasar del tiempo ha ganado notoriedad repensar en el cuidado del ambiente (Salas et al., 2020; Sun et at., 2018). Latinoamérica no es ajena a esta problemática, ya que existe una degradación progresiva en el aire y suelo, que viene afectando considerablemente el medio ambiente (Ospina, 2019).

En las últimas décadas, diversas investigaciones en áreas como la economía, ética, sociología, psicología, derecho o política, se vienen enfocando en la responsabilidad social (RS). Debido a los problemas que se viene ocasionando como es la contaminación ambiental, por lo que diversas organizaciones promueven prácticas ProPaz en los cuales deben involucrarse diseños basados en la RS (Perdomo, 2017).

Siendo que los hombres y mujeres son actores sociales que viven y construyen sus realidades ya sea por su lenguaje, creencias, observación, subjetividad, respetando su entorno natural (López y Cabrera, 2021), la visión debe ser de manera conjunta enfocándose en intereses comunes, como es el respeto del medio ambiente, viéndose reflejado en el acciones del ser humano, mostrando una buena sinergia ecológica (Viorel, 2019); la RS social no solo se aplica en el sector privado sino también en otros campos y es en las instituciones estatales como se ha dado en estos últimos años con una mayor relevancia en los individuos (Rakan et al., 2020).

Otro tema muy importante que está relacionado con la RS es la educación ambiental que busca en los seres humanos establecer vínculos entre los integrantes de una sociedad mediante el conocimiento y promoviendo conductas que contribuyan al respeto del medio ambiente (Pulido y OIivera, 2018; Gallardo et al., 2020), por otro las organizaciones al desarrollar sus actividades tienen el compromiso de ser ecosostenibles para no alterar ni dañar al ecosistema (Lara et al., 2020) 
Otro problema que se presentó fue el coronavirus COVID-19, conociéndose al inicio del primer mes del año pasado en el país chino, expandiéndose a nivel mundial y el 11 de marzo fué declarado organización mundial de la salud (OMS), presentándose un problema adicional que ayudó a agudizar la contaminación, observándose en las calles mayor acumulamiento de residuos sólidos como son las mascarillas, protectores faciales y guantes (He y Harris; Mohammad, 2019)

En el Perú la responsabilidad social se ha dado en diversas etapas, teniendo como inicio desde algunos conceptos de filantropía hasta la aplicación en la vida diaria. El compromiso que tenemos los peruanos está referido a conservar, respetar y amar nuestro medio ambiente, para que las futuras generaciones lo disfruten, para ello hay que estar conscientes de las decisiones que tomamos. Según (Organización Mundial del Turismo ,2018) (OMT) en el Perú se respira mal, ya que se encuentra ubicado en el décimo primero de los más contaminados, antecedido por Kazajistán y seguido por Etiopia.

Así mismo, en Lima han fallecido en los últimos años aproximadamente quince mil ciudadanos debido a diferentes complicaciones, enfermedades respiratorias y cardiacas ocasionadas por la contaminación ambiental, la idiosincrasia de una parte de la población es muy grande, pues no toma conciencia de sus acciones y como consecuencias genera contaminación en el medio ambiente (OMS, 2019). Según Ley 28611 (Ministerio del ambiente ,2012) establece los ejes estratégicos ambientales, según el Organismo de Evaluación y Fiscalización Ambiental, (2018) identificó 92 distritos a nivel nacional que requieren de atención inmediata mejorar la gestión de sustancias sólidas y sus servicios de limpieza, estando en la lista en la provincia de Huaral. Donde se ha observado que existe carencia de la educación ambiental en la provincia, conllevando a estos problemas que afectan en el entorno natural (Barrios, 2018; Činčera et al., 2020; Genc et al., 2001)

El problema que se ha presentado es el acumulamiento de la basura en distintas partes de Atavillos bajo, especialmente en la comunidad de Florida donde se genera un aspecto no apropiado para el mismo poblador, además con esta coyuntura presentada en este tiempo como es el "Covid-19", ha permitido que se genere mayor desorden y acumulación viéndose reflejado en el malestar entre sus miembros, y aquellas personas que visitan el lugar afectando a la población con posibles enfermedades, no hay un plan 
de desarrollo, la crisis de salud producto de Covid-19 nos ha llevado a reflexionar y ser mejores personas para contribuir a la sociedad (Severino et al 2020)

Se consideró la necesidad de hacer un estudio ya que hasta la fecha está en abandono por parte de sus autoridades, también mencionar en este distrito se encuentra uno de los atractivos turísticos llamado Machu Picchu Limeño o Ciudad de Fuego, sitio ceremonial que data de tiempos preincaicos y fue reconocido por el (INC,1999) con RDN. №283/INC como patrimonio peruano, siendo vital para el desarrollo del turismo que busca generar divisas contribuyendo al PBI nacional.

La aplicación del enunciado holopráxico, permitió precisar lo que se necesitó conocer de manera concreta el problema presentado (Hurtado, 2015). En tal sentido, el problema general quedó formulado en los siguientes términos: ¿Cómo la RS incide en la gestión ambiental en el distrito de Atavillos Bajo, año 2021?, las interrogantes específicas: ¿Cómo la RS incide en la evaluación del impacto ambiental?, ¿Cómo la RS incide en la auditoría ambiental?, ¿Cómo la RS incide en el análisis del ciclo de vida?, y finalmente ¿Cómo la RS incide en el sistema de gestión ambiental?

El trabajo de investigación se justifica según la tipología de Fernández-Bedoya (2020) y Vico (2018) permite dar a conocer la importancia de la investigación desarrollada, considerando su relevancia práctica-social, metodológica, teórica y epistemológica. Es así que la justificación teórica se ha basado en describir las definiciones de responsabilidad social, así también en gestión ambiental para conocer el alcance del contexto actual y la trascendencia en el campo de la administración.

Justificación Práctica -Social, se aportó en la elaboración de un plan para capacitar a la población sobre temas relacionados al medio ambiente, buscando la concientización en cada miembro, se considera que será de gran ayuda, pero también depende del compromiso de los pobladores de Florida.

En cuanto, justificación metodológica Fernández-Bedoya (2020) se aportó con la construcción de dos cuestionarios de cada variable, teniendo en cuenta el contexto actual, asimismo tomando en cuenta los criterios de validéz de contenido como juicio de expertos-método del consenso grupal, validez de constructo mediante la aplicación del análisis factorial y finalmente se realizó la confiabilidad con el coeficiente Alfa de Cron Bach. 
Y Como justificación epistemológica, el estudio se ha basado en el positivismo y empirismo con respecto a la medición controlada de los datos obtenidos, los cuales fueron sometidos a un proceso exploratorio inferencial deductivo, manteniendo distancia con el objeto de estudio y la posición del investigador fue neutral (Lavado, 2020)

Es por ello que el objetivo de investigación quedó redactado siguiendo las orientaciones de Hurtado (2015), a decir el objetivo general: Demostrar cómo la RS incide en la gestión ambiental, y en cuanto a los objetivos específicos en los siguientes términos: Demostrar cómo la RS incide en la evaluación del impacto ambiental, Demostrar cómo la RS incide en la auditoría ambiental, demostrar cómo la RS incide en el análisis del ciclo de vida, y finalmente cuarto objetivo específico: Demostrar cómo la RS incide en el sistema de gestión ambiental.

Respecto a la formulación de las hipótesis, se siguió las recomendaciones de Amaiquema (2019). En tal razón la hipótesis general se describe en los siguientes términos: la RS incide significativamente en la gestión ambiental en el distrito de Atavillos Bajo, en el año 2021. Y la redacción de sus hipótesis específicas: la RS incide significativamente en la evaluación del impacto ambiental, RS incide en la auditoría ambiental, la RS incide significativamente en el análisis del ciclo de vida, finalmente, la RS incide significativamente en el sistema de gestión ambiental.

\section{ESTRATEGIAS METODOLÓGICAS O MATERIALES Y MÉTODOS}

\section{Tipo y diseño de investigación}

Según CONCYTEC (2018) mediante la ley $\mathrm{N}^{\circ} 30806$ refiere que la investigación científica debe ser legítimo y busca alcanzar nuevo conocimiento. Asimismo, el paradigma aplicado en la investigación fue el Positivista, con una investigación presentada ha sido básica, cuya realidad puede ser medible (Pacori, 2019; Naupas et al., 2013)

Se utilizó un enfoque cuantitativo, así también se ha utilizado la recolección y análisis de datos para poder dar respuesta a las interrogantes preguntas de investigación con ello se ha podido realizar la prueba de hipótesis que fueron establecidas con antelación confiando en la medición numérica, para ello se tuvo que usar la estadística y establecer con exactitud a la población (Gómez,2012, p.33) 
Para Hernández y Mendoza, (2005) "En la ciencia social que tuvo origen en la obra Augusto Comte (1798-1857), así también Emile Durkheim (1858-19179), mencionan que en el estudio de actos influyentes debe poder aplicarse un solo mismo método, el científico (p.5). Se Necesita tener un espacio preciso al cual arribar (planteamiento especificado y delimitado) y un mapa preciso (Hernández y Mendoza, 2018, p.7)

La investigación es de tipo básica, porque la investigación se realizó para obtener conocimientos, además porque se fundamenta en la teoría y busca recoger información de la realidad y poder nutrir el conocimiento científico, identificando el problema, para dar propuestas de solución. El nivel explicativo, porque está enfocado en establecer la causalidad, dando mayor énfasis en poder evidenciar, demostrar, probar su interés en lo que ocurre en el fenómeno y saber la condición en que se encuentra (Pacori 2019, p, 75). El diseño no experimental-transversal-causal, porque nuestra variable se mantuvo como tal, basándonos en la observación de fenómeno como este se presenta en un entorno natural, siendo después analizados de manera minuciosa, también el instrumento fue aplicado en su solo momento en el distrito (Pacori 2019). El Método Hipotético deductivo, porque en nuestra investigación se plantea un problema a través de la observación y análisis del mismo en un caso específico o particular (Pacori,2019).

\section{Figura 1}

\section{Esquema del diseño $x_{1} \longrightarrow r_{1}$}

Donde:

$\mathrm{X}_{1}$ : Variable independiente: Responsabilidad social

$\mathrm{Y}_{1}$ : Variable dependiente: Gestión ambiental

Fuente: Elaboración propia.

\section{Variables y operacionalización}

La variable independiente fue la R.S su definición conceptual es presentada por los autores Ocampo y Rivera (2017) es el actuar ético de manera estratégico de las empresas y debe estar plasmada en la misma visión que tienen y en su vida diaria $(\mathrm{p}, 33)$.

Definición operacional, midió la percepción de los pobladores del distrito de Atavillos bajo sobre la variable independiente responsabilidad social, mediante la aplicación de un cuestionario cuya escala fue de medición ordinal del 1 al 5. Siendo sus dimensiones según la ISO 26000 (2010): Derechos humanos, prácticas laborales, practicas justas de 
operación, asuntos consumidores, desarrollo sostenible, participación dinámica de manera integral en la comunidad. Respecto a la variable dependiente de gestión ambiental según

Rojo et al., (2018) refiere que la GA es un acumulado de gestiones enfocados a lograr de manera efectiva y razonablemente en el proceso de decisiones concerniente a la preservación, salvaguardia, amparo y florecimiento del entorno natural, y se enfoca en una sincronización multidisciplinar en una participación conjunta.

Definición operacional, midió la percepción de los pobladores del distrito de Atavillos bajo, sobre la variable dependiente Gestión ambiental, mediante la aplicación de un cuestionario cuya escala fué de medición ordinal del 1 al 5. Según la ISO 14001(2015) Evaluación del impacto ambiental, auditoría ambiental, análisis del ciclo de vida y sistema de gestión ambiental

\section{Población, muestra y muestreo}

"La población es un conjunto total de individuos, objetos o medidas con características comunes observables, limitado, en un determinado momento y espacio geográfico" (Pacori, 2019). La población total estuvo conformada por los pobladores de la comunidad de Florida perteneciente al distrito de Atavillos bajo, provincia de Huaral del año 2021, el cual ascendió a un total 84, los mismos que su totalidad han sido encuestados por tal razón no se han utilizado ninguna técnica de muestreo (Hernández et al., 2014)

\section{Técnicas e instrumentos de recolección de datos}

Se ha realizado la recolección de datos a través de la encuesta como técnica teniendo un cúmulo de ítems del cuestionario con una escala de Likert, obteniéndose información sobre el análisis de estudio Pacori (2019). Asimismo, precisar que no hay una contestación buena o mala debido que se busca conocer la percepción del encuestado según las variables estudiadas. Los instrumentos de las variables R.S y la G.A se han construido en su totalidad, siendo sometidos a un proceso de validéz y confiablidad.

Referente a la validez se ha citado a cuatro profesionales con una amplia experiencia en investigación y con la información dada de los tres elementos que se consideraron (pertinencia relevancia y claridad) se ha procesado los datos de contenido con la prueba binomial obteniendo el siguiente resultado 0.0002441 el valor obtenido es menor a 0.05 se puede decir el instrumento válido y puede aplicarse. 
Según Hurtado (2013) menciona sobre la importancia de poder hacer uso de la validez de constructo a través del análisis factorial utilizando el estadígrafo KMO y Bartlett pero no se obtuvo debido el puntaje deseado, para ello se procedió a aplicar el estadígrafo Rho de Spearman considerando como referencia un puntaje total de la variable así también con la obtención de puntaje de cada dimensión con la finalidad de medir la solidez del instrumento. Para la confiabilidad se aplicó una muestra piloto de 20 participantes y se obtuvo el coeficiente de Alfa de Cronbach de la primera variable 0.745 y 0.81 de la segunda variable por tanto ambos instrumentos son considerados aceptables y de una alta categoría (Pacori, 2019)

\section{Procedimientos}

Se ha iniciado con la elaboración de la carta de presentación para pedir el respectivo permiso del estudio realizado, dándose un acercamiento con el alcalde vía telefónica y después de forma presencial en la ciudad de Huaral, de esa misma manera se tuvo contacto con el presidente de la comunidad de Florida mediante llamada telefónica y después in situ. Se digitó los cuestionarios en Word, debido a que el estudio a realizar ha sido en una comunidad rural y las razones era porque había limitaciones como el poco acceso a internet e incluso el desconocimiento sobre el manejo de la tecnología de información, es así que se realizó la visita in situ en la comunidad respetando los debidos protocolos de seguridad para recolectar la información, seguidamente se ha procedido a sistematizar, después a pasó a una ahoja de MS Excel, seguido se importó al SPSS para su respectivo análisis.

\section{Método de análisis de datos}

Se llegó a sistematizar los datos provenientes de la aplicación de las encuestas, en el programa MS Excel con la finalidad de ordenar la información y trasladarla para ordenar a la de vista de datos del programa SPSS v26, en el cual se realizó el análisis descriptivo de la distribución de frecuencias de cada una de las variables con sus respectivas dimensiones, asimismo la representación visual a graves del gráfico de barras. Para el análisis inferencial se ha utilizado la regresión logística ordinal, de esta manera se determinó su nivel de incidencia para luego hacer la contrastación de las hipótesis generales y específicas (Rustom, 2012).

\section{Aspectos éticos}


Según Palencia y Ben (2013), establecieron cual son los lineamientos normativos, que dio origen a la averiguación se establecieron reglas normativas, que dieron origen a respetar con lo estipulado, se trabajó de manera minuciosa, con mucha responsabilidad respetando la autonomía de elementos muestrales toda vez que tenían la posibilidad de decidir no participar en el estudio, y compromiso de toda la información recopilada sin realizar ninguna discriminación de la información recopilada, además se llevó a cabo correctamente el proceso informando a los participantes que el uso de dicha información es únicamente con fines académicos. La investigación es de autoría, a su vez tomando como referencias bibliográficas respectivas procedentes de artículos científicos, tesis, libros, normas y leyes, siendo obtenida se citó junto con sus autores considerando el Manual de la APA en su séptima edición, lo cual implica el respecto a la producción intelectual, luego la investigación tuvo un proceso para medir la similitud procesada a través del turnitín (Hortal, 2002).

\section{RESULTADOS Y DISCUSIÓN}

\section{Resultados}

\section{Tabla 1}

Distribución de frecuencias de la responsabilidad social

\begin{tabular}{llcccc}
\hline & Frecuencia & Porcentaje & $\begin{array}{c}\text { Porcentaje } \\
\text { válido }\end{array}$ & $\begin{array}{c}\text { Porcentaje } \\
\text { acumulado }\end{array}$ \\
\hline \multirow{3}{*}{ Válido } & Deficiente & 42 & 50,0 & 50,0 & 50,0 \\
& Moderado & 40 & 47,6 & 47,6 & 97,6 \\
& Eficiente & 2 & 2,4 & 2,4 & 100,0 \\
& Total & 84 & 100,0 & 100,0 & \\
\hline \multicolumn{5}{r}{ Fuente: base de datos (ver anexo) }
\end{tabular}

\section{Figura 1}

Niveles de la R.S

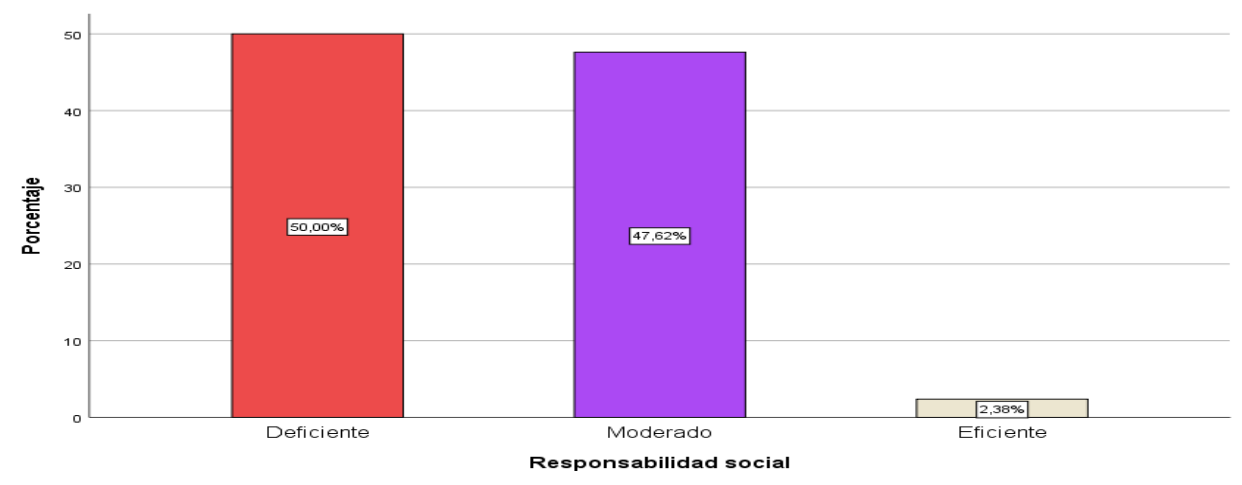


De los resultados mostrados en la tabla 1 y figura 2 muestra que el $50 \%$ de los pobladores del distrito, manifiestan que la responsabilidad social es nivel deficiente, asimismo. Mientras que el 47,62\% refiere que el nivel es moderado. También, el 2,38\% menciona que el nivel es muy eficiente. Así mismo, ante la prevalencia de los datos presentados en tabla y figura podemos manifestar sobre la responsabilidad social es deficiente en la gestión ambiental. Atavillos Bajo, Lima, 2021.

\section{Tabla 2}

Distribución de frecuencias de las dimensiones de la responsabilidad social

\begin{tabular}{|c|c|c|c|c|c|c|c|c|c|c|c|c|}
\hline \multirow[t]{2}{*}{ Nivel } & \multicolumn{2}{|c|}{$\begin{array}{l}\text { Derechos } \\
\text { humanos }\end{array}$} & \multicolumn{2}{|c|}{$\begin{array}{l}\text { Prácticas } \\
\text { laborales }\end{array}$} & \multicolumn{2}{|c|}{$\begin{array}{l}\text { Prácticas } \\
\text { Justas de } \\
\text { Operación }\end{array}$} & \multicolumn{2}{|c|}{$\begin{array}{l}\text { Asuntos de } \\
\text { consumidores }\end{array}$} & \multicolumn{2}{|c|}{$\begin{array}{c}\text { Desarrollo } \\
\text { sostenible }\end{array}$} & \multicolumn{2}{|c|}{$\begin{array}{c}\text { Participación } \\
\text { activa y } \\
\text { desarrollo }\end{array}$} \\
\hline & $\mathbf{f}$ & $\%$ & $\mathbf{F}$ & $\%$ & $\mathbf{F}$ & $\%$ & f & $\%$ & $\mathbf{F}$ & $\%$ & f & $\%$ \\
\hline Deficiente & 60 & 71.4 & 39 & 46.4 & 62 & 73.8 & 49 & 58.3 & 58 & 69.0 & 16 & 19.0 \\
\hline Moderado & 23 & 27.4 & 37 & 44.0 & 19 & 22.6 & 33 & 39.3 & 21 & 25.0 & 38 & 45.2 \\
\hline Eficiente & 1.0 & 1.2 & 8.0 & 9.5 & 3.0 & 3.6 & 2.0 & 2.4 & 5 & 6.0 & 30 & 35.7 \\
\hline Total & 84 & 100 & 84 & 100 & 84 & 100 & 84 & 100 & 84 & 100 & 84 & 100 \\
\hline
\end{tabular}

Fuente: base de datos (ver anexo)

\section{Figura 3}

Niveles según dimensiones de la responsabilidad social

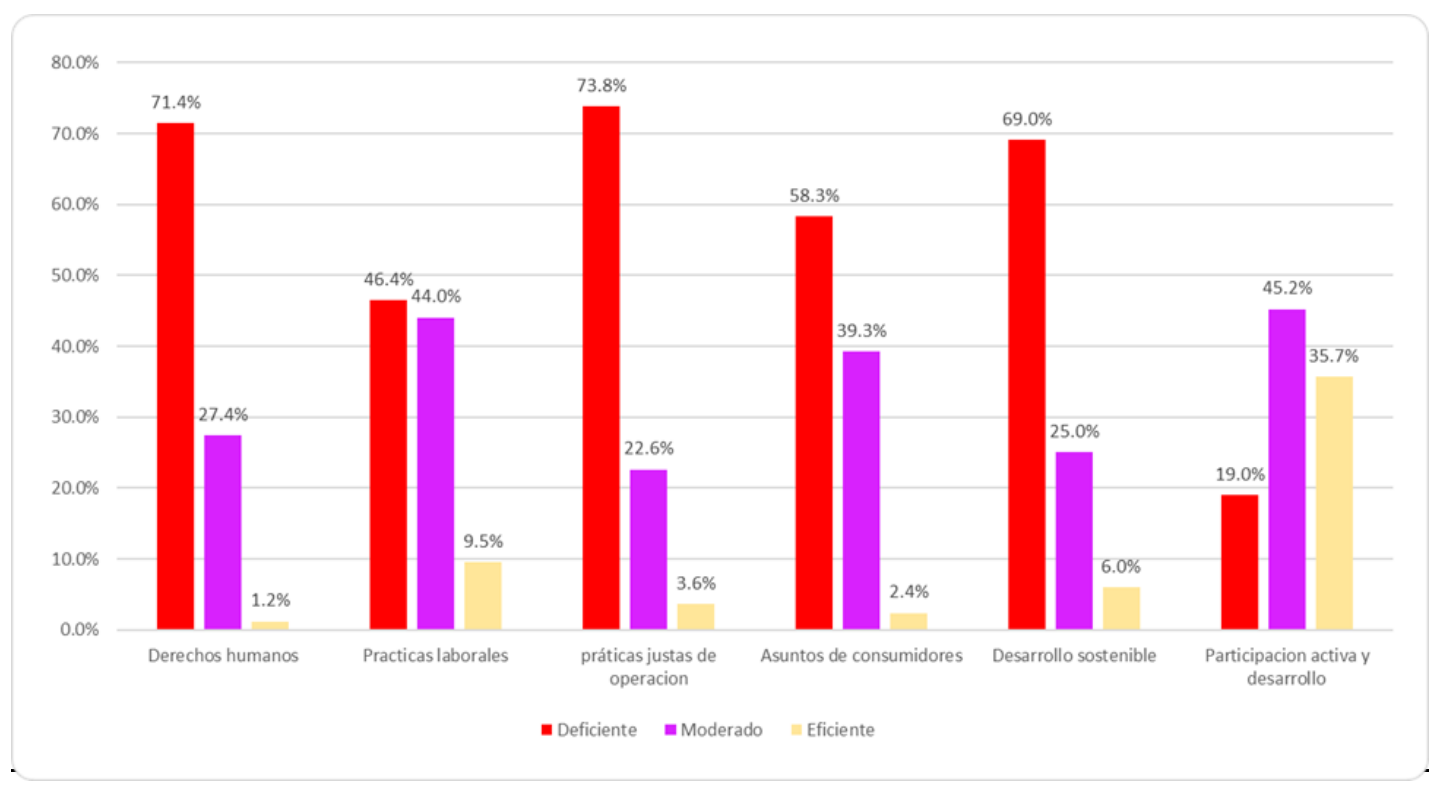

De la tabla 2 y figura 3, se observa el $71.4 \%$ de los encuestados tiene la percepción de que la dimensión derechos humanos es de nivel deficiente, el $46.4 \%$ menciona que la 
dimensión prácticas laborales es de nivel deficiente, como también manifiestan que en la dimensión practicas justas de operación con un $73.8 \%$ con un nivel deficiente por otro lado un $58.3 \%$ respectivamente percibe en la dimensión asuntos de consumidores un nivel deficiente, en el caso de la dimensión desarrollo sostenible de los encuestados se muestra un $69 \%$ con un nivel deficiente.

Además podemos visualizar, que en un porcentaje promedio del $45.2 \%$, los pobladores encuestados perciben que la responsabilidad social en cada una de sus dimensiones es de moderado, presentándose el más alto porcentaje, seguida por la dimensión práctica laborales con un $44.0 \%$, así mismo con un $39.3 \%$ asuntos de consumidores, por otro lado con un $35.7 \%$ participación activa y desarrollo, también un $27.4 \%$ derechos humanos, seguido de $25.0 \%$ desarrollo sostenible y un $22.6 \%$ prácticas justas de operación.

Sin embargo, un grupo equivalente a un porcentaje menor al $10 \%$ refieren un nivel muy eficiente en cuanto a la responsabilidad social, como se presenta, que un $1.2 \%$ refiere este nivel en la dimensión derechos humanos, también que el 9.5\% en la dimensión prácticas laborales, mientras que el 3.6\% en la dimensión prácticas justas de operación, asimismo que el $2.4 \%$ en la dimensión asuntos de consumidores, además que un $6.0 \%$ en la dimensión desarrollo sostenible y finalmente que el 19\% en la dimensión participación activa y desarrollo según la percepción de los pobladores del distrito de Atavillos Bajo, 2021.

\section{Resultados descriptivos de gestión ambiental}

\section{Tabla 3}

Distribución de frecuencias de la gestión ambiental

\begin{tabular}{llcccc}
\hline & Frecuencia & Porcentaje & $\begin{array}{c}\text { Porcentaje } \\
\text { válido }\end{array}$ & $\begin{array}{c}\text { Porcentaje } \\
\text { acumulado }\end{array}$ \\
\hline Válido & Deficiente & 65 & 77,4 & 77,4 & 77,4 \\
& Moderado & 18 & 21,4 & 21,4 & 98,8 \\
& Eficiente & 1 & 1,2 & 1,2 & 100,0 \\
& Total & 84 & 100,0 & 100,0 & \\
\hline
\end{tabular}

Fuente: base de datos (ver anexo) 


\section{Figura 4}

Niveles de la gestión ambiental

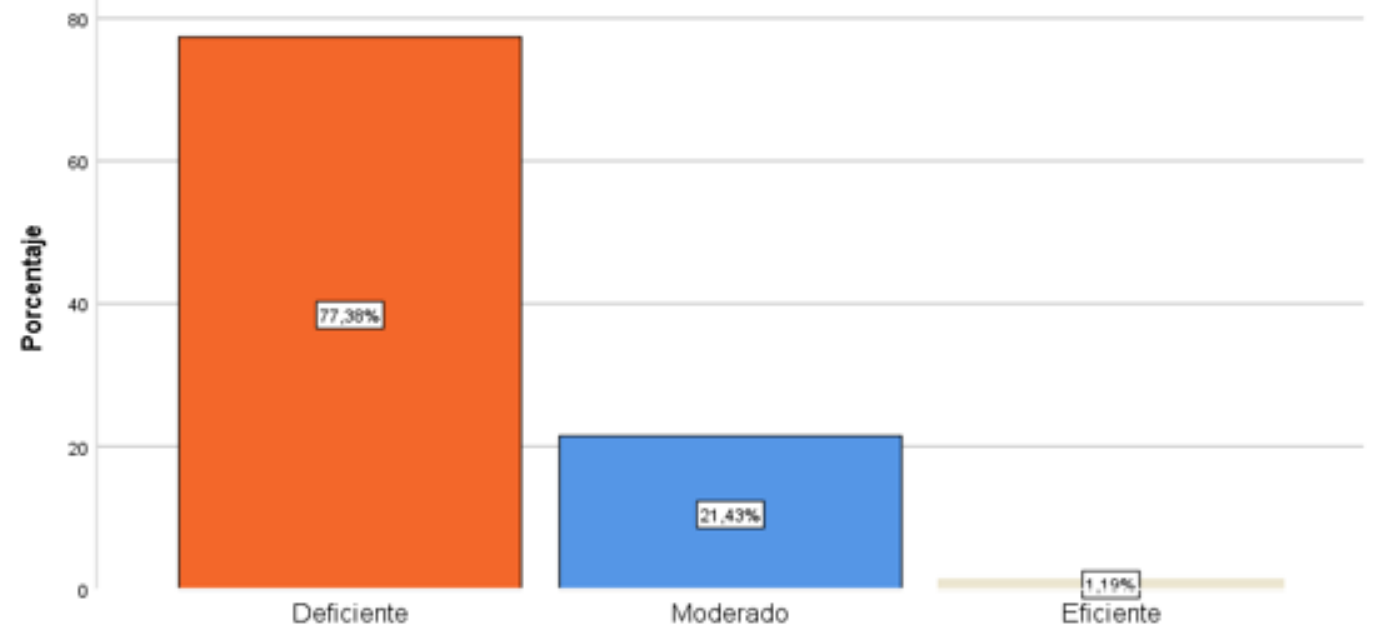

De los resultados mostrados en la tabla 3 y figura 4 muestra que el $77.38 \%$ de los pobladores del distrito de Atavillos bajo, manifiestan sobre la gestión ambiental es nivel deficiente, Mientras que, el 21,43\% refiere que el nivel es moderado. También, el 1,19\% menciona que el nivel es muy eficiente. Así mismo, ante la prevalencia de los datos presentados en tabla y figura podemos manifestar que la gestión ambiental es deficiente.

\section{Tabal 4}

Distribución de frecuencias según las dimensiones de la gestión ambiental

\begin{tabular}{|c|c|c|c|c|c|c|c|c|}
\hline \multirow[t]{2}{*}{ Nivel } & \multicolumn{2}{|c|}{$\begin{array}{l}\text { Evaluación } \\
\text { del impacto } \\
\text { ambiental }\end{array}$} & \multicolumn{2}{|c|}{$\begin{array}{l}\text { Auditoría } \\
\text { ambiental }\end{array}$} & \multicolumn{2}{|c|}{$\begin{array}{l}\text { Análisis del } \\
\text { ciclo de vida }\end{array}$} & \multicolumn{2}{|c|}{$\begin{array}{c}\text { Sistema de gestión } \\
\text { ambiental }\end{array}$} \\
\hline & $\mathbf{F}$ & $\%$ & f & $\%$ & $\mathbf{F}$ & $\%$ & $\mathbf{F}$ & $\%$ \\
\hline Deficiente & 60 & 71.4 & 25 & 29.8 & 49 & 58.3 & 78 & 92.9 \\
\hline Moderado & 22 & 26.2 & 26 & 31.0 & 32 & 38.1 & 5 & 6.0 \\
\hline Eficiente & 2 & 2.4 & 33 & 39.3 & 3 & 3.6 & 1 & 1.2 \\
\hline Total & 84 & 100 & 84 & 100 & 84 & 100 & 84 & 100 \\
\hline
\end{tabular}

Fuente: base de datos (ver anexo) 


\section{Figura 5}

Niveles según dimensiones de la gestión ambiental

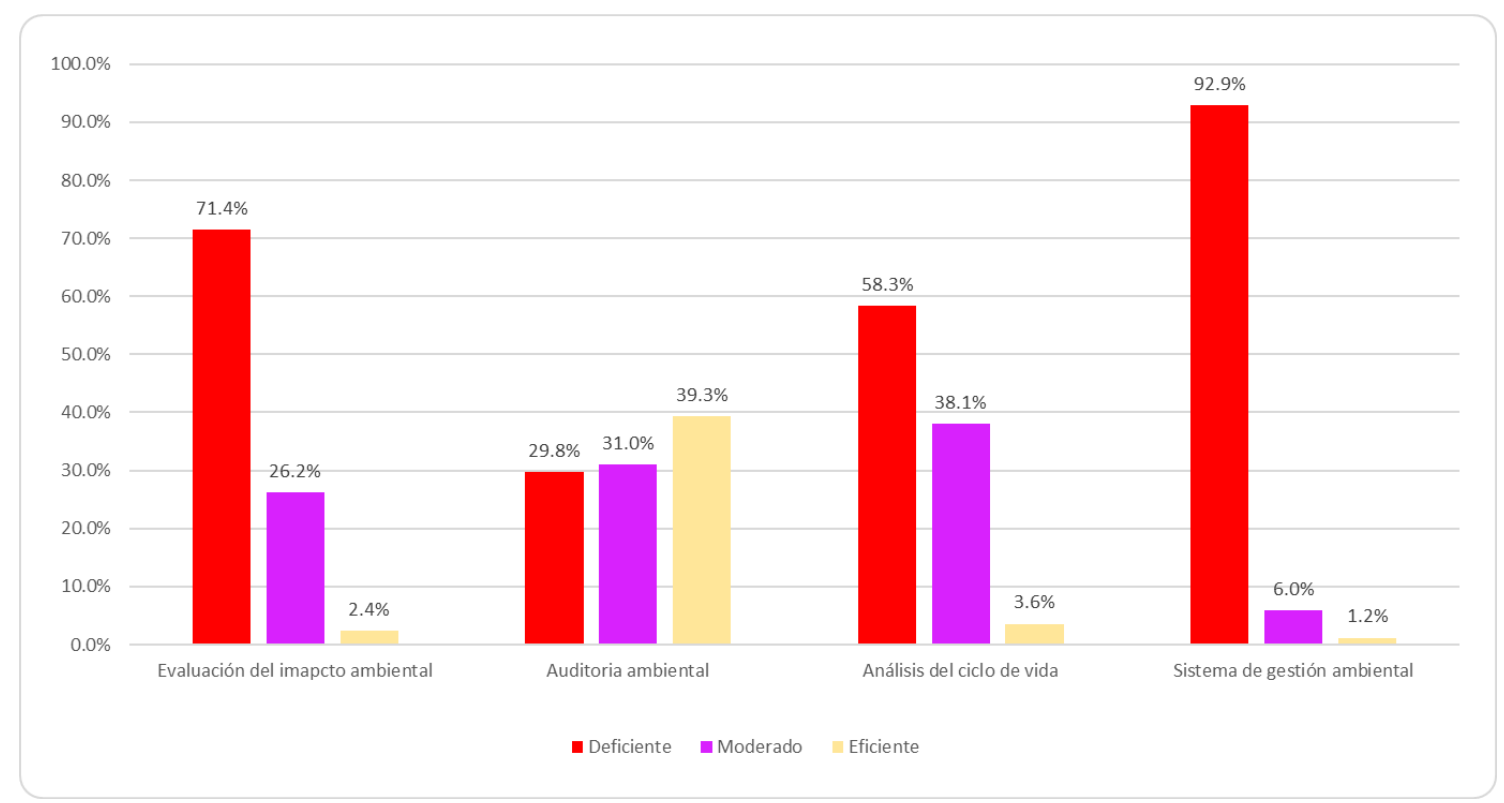

De la tabla 4 y figura 5, se observa el $71.4 \%$ de los encuestados tiene la percepción de que la dimensión evaluación del impacto ambiental es de nivel deficiente, el 39.3\% menciona que la dimensión auditoría ambiental es de nivel eficiente, como también manifiestan que en la dimensión análisis del ciclo de vida con un $58.3 \%$ con un nivel deficiente, y un $92.9 \%$ respectivamente percibe en la dimensión sistema de gestión ambiental con un nivel deficiente. Podemos visualizar, que en un porcentaje promedio de los pobladores encuestados perciben que la gestión ambiental en cada una de sus dimensiones es de nivel moderado, presentándose el más alto porcentaje (38.1\%) en la dimensión análisis del ciclo de vida, mientras que la auditoría ambiental con un $31.0 \%$, así mismo con un $26.2 \%$ evaluación del impacto ambiental y un $6.0 \%$ sistema de gestión ambiental. Sin embargo, un grupo equivalente a un porcentaje menor al $29.8 \%$ refieren un nivel deficiente en cuanto a la gestión ambiental, como se presenta, que un $29.8 \%$ refiere este nivel en la dimensión auditoría ambiental, también que el 3.6\% en la dimensión análisis del ciclo de vida, mientras que el $2.4 \%$ en la dimensión evaluación del impacto ambiental, y un $1.2 \%$ en la dimensión de sistema de gestión ambiental desarrollo según la percepción de los pobladores del distrito 


\section{Resultados inferenciales}

\section{Prueba de hipótesis general}

Prueba de hipótesis general

$\mathbf{H}_{0}$ : La responsabilidad social no incide de modo significativo en la gestión ambiental. Atavillos bajo, Lima 2021.

H1: La responsabilidad social incide de modo significativo en la gestión ambiental Atavillos bajo, Lima 2021.

Nivel de significancia: $\alpha=5 \%$.

Estadístico de prueba: Regresión logística Ordinal (RLO).

Regla de decisión: Si: p-valor $<0.05$, se rechaza la hipótesis nula, y el contraste es significativo.

\section{Tabla 5}

Determinación del ajuste de la R.S que incide en la gestión ambiental

\begin{tabular}{lcccc}
\hline \multicolumn{1}{c}{ Modelo } & $\begin{array}{c}\text { Logaritmo de la } \\
\text { verosimilitud -2 }\end{array}$ & Chi-cuadrado & Gl & Sig. \\
\hline Sólo intersección & 39,064 & & 1 &, 000 \\
Final & 8,868 & 30,196 & 1 & \\
\hline
\end{tabular}

De acuerdo a los resultados de la tabla 5 se observa que el valor de la significancia $0,000<0,05$ por lo tanto se rechaza la hipótesis nula y se acepta la hipótesis alterna, es decir la responsabilidad social incide de modo significativo en la gestión ambiental, Atavillos bajo, Lima 2021.

\section{Tabla 6}

Pseudo coeficiente de determinación de las variables para la hipótesis general

\begin{tabular}{ll}
\hline Cox y Snell &, 302 \\
Nagelkerke &, 439 \\
McFadden &, 309 \\
\hline
\end{tabular}

En la tabla 6 se observa según el valor del Pseudo R cuadrado de Nagelkerke, que la responsabilidad social incide en un $43.9 \%$ en la gestión ambiental, Atavillos bajo, Lima 2021. 


\section{Tabla 7}

Estimaciones de parámetros para la hipótesis general

\begin{tabular}{lllllllll}
\hline & & $\begin{array}{c}\text { Estimaci } \\
\text { ón }\end{array}$ & $\begin{array}{c}\text { Desv. } \\
\text { Error }\end{array}$ & Wald & gl & Sig. & \multicolumn{2}{c}{$\begin{array}{c}\text { Intervalo de } \\
\text { confianza al 95\% }\end{array}$} \\
\hline Umbral & $\begin{array}{l}\text { Límite } \\
\text { inferior }\end{array}$ & $\begin{array}{c}\text { Límite } \\
\text { superior }\end{array}$ \\
& $\begin{array}{l}\text { [GA_ } \\
\text { Deficiente] } \\
{[\mathrm{GA}}\end{array}$ & 8,147 & 2,203 & 13,684 & 1 &, 000 & 3,831 & 12,464 \\
& $\begin{array}{l}\text { Moderado] } \\
\text { Ubicación }\end{array}$ & 12,509 & 3,006 & 17,324 & 1 &, 000 & 6,619 & 18,400 \\
\hline
\end{tabular}

En la tabla 7, los valores de las significancias tanto para el nivel deficiente como el nivel moderado de la gestión ambiental (variable dependiente) fueron 0,000, lo cual cumple con la condición de ser menor a 0,05; asimismo, los coeficientes Wald fueron 13,684 y 17,324 respectivamente, lo cual cumple con la condición de ser mayor a 4; por lo tanto, estos niveles (deficiente y moderado) fueron incididos de modo significativo por la responsabilidad social (variable independiente).

Por consiguiente, se rechazó Ho y aceptó H1.

Prueba de hipótesis especificas

\section{Prueba específica 1}

Ho: La responsabilidad social no incide de modo significativo en la evaluación del impacto ambiental. Atavillos bajo, Lima 2021.

H1: La responsabilidad social incide de modo significativo en la evaluación del impacto ambiental. Atavillos bajo, Lima 2021.

\section{Tabla 8}

Determinación del ajuste de la responsabilidad social incide en la evaluación del impacto ambiental

\begin{tabular}{lccccc}
\hline \multicolumn{1}{c}{ Modelo } & $\begin{array}{c}\text { Logaritmo de la } \\
\text { verosimilitud -2 }\end{array}$ & Chi-cuadrado & Gl & Sig. \\
\hline Sólo intersección & 25,861 & & 1 &, 002 \\
Final & 15,880 & 9,981 & 1 & \\
\hline
\end{tabular}

Según la tabla 8 se observa que la significancia es igual a $0,002<$ a 0,05 por lo tanto se rechaza la hipótesis nula y se acepta la hipótesis alterna, es decir la responsabilidad 
social incide de modo significativo en la evaluación del impacto ambiental. Atavillos bajo, Lima 2021.

\section{Tabla 9}

Pseudo coeficiente de determinación de las variables para la primera hipótesis

\begin{tabular}{lc}
\hline Cox y Snell &, 112 \\
Nagelkerke &, 151 \\
McFadden &, 087
\end{tabular}

En la tabla 9 se observa según el valor del Pseudo R cuadrado de Nagelkerke, que la responsabilidad social incide en un $15.1 \%$ en la evaluación del impacto ambiental, Atavillos bajo, Lima 2021.

\section{Tabla 10}

Estimaciones de parámetros para la hipótesis específica 1

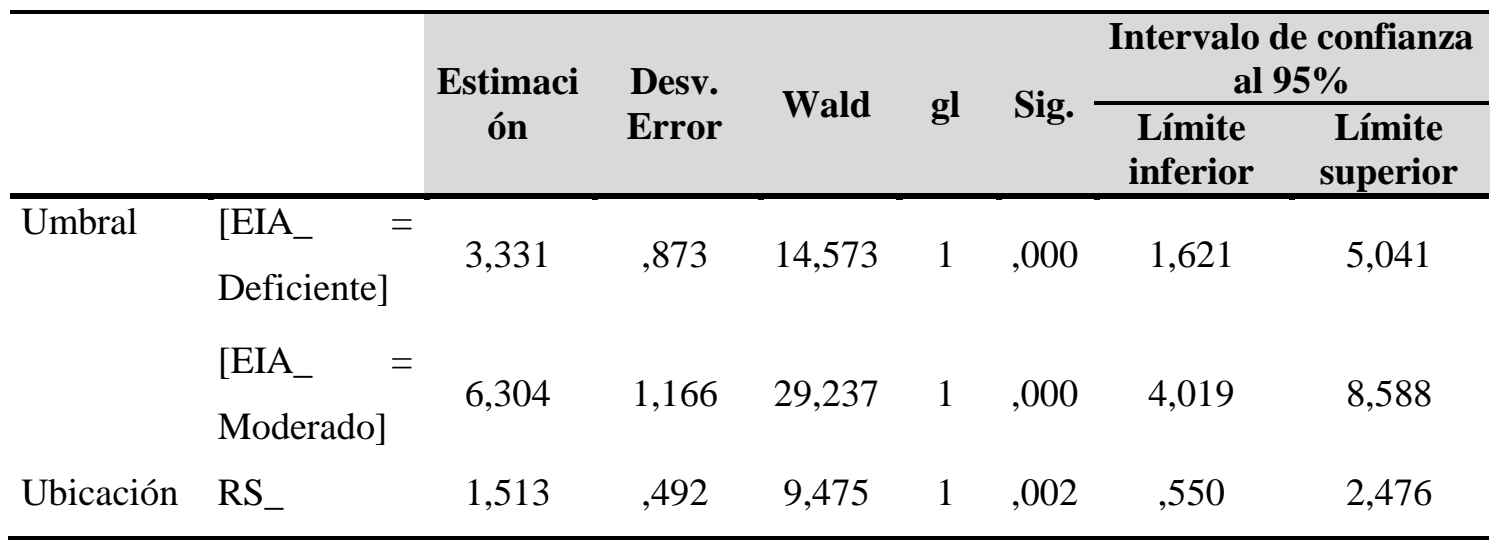

$\overline{\text { En la tabla 10, los valores de las significancias tanto para el nivel deficiente como el }}$ nivel moderado de la evaluación del impacto ambiental (variable dependiente) fueron 0,000, lo cual cumple con la condición de ser menor a 0,05; asimismo, los coeficientes Wald fueron 14,573 y 29,237 respectivamente, lo cual cumple con la condición de ser mayor a 4; por lo tanto, estos niveles (deficiente y moderado) fueron incididos de modo significativo por la responsabilidad social (variable independiente).

Por consiguiente, se rechazó Ho y aceptó H1.

Por otro lado, la prueba específica 2 se tiene que.

Ho: La responsabilidad social no incide de modo significativo en la auditoría ambiental. Atavillos bajo, Lima 2021.

H1: La responsabilidad social incide de modo significativo en la en la auditoría ambiental. Atavillos bajo, Lima 2021. 


\section{Tabla 11}

Prueba de ajuste del modelo y Pseudo $R$ cuadrado de la responsabilidad social en la segunda hipótesis

\begin{tabular}{lcccc}
\hline \multicolumn{1}{c}{ Modelo } & $\begin{array}{c}\text { Logaritmo de la } \\
\text { verosimilitud -2 }\end{array}$ & Chi-cuadrado & Gl & Sig. \\
\hline $\begin{array}{l}\text { Sólo intersección } \\
\text { Final }\end{array}$ & 34,296 & & 1 &, 000 \\
\hline
\end{tabular}

De acuerdo a los resultados de la tabla 11 se observa que el valor de la significancia $0,000<0,05$ por lo tanto se rechaza la hipótesis nula y se acepta la hipótesis alterna, es decir la responsabilidad social incide de modo significativo en la auditoría ambiental, Atavillos bajo, Lima 2021.

\section{Tabla 12}

Pseudo coeficiente de determinación de las variables para segunda hipótesis

\begin{tabular}{lc}
\hline Cox y Snell &, 167 \\
Nagelkerke &, 189 \\
McFadden &, 084
\end{tabular}

En la tabla 12 se observa según el valor del Pseudo R cuadrado de Nagelkerke, que la responsabilidad social incide en un $18.9 \%$ en la auditoría ambiental, Atavillos bajo, Lima 2021

\section{Tabla 13}

Estimaciones de parámetros para la hipótesis específica 2

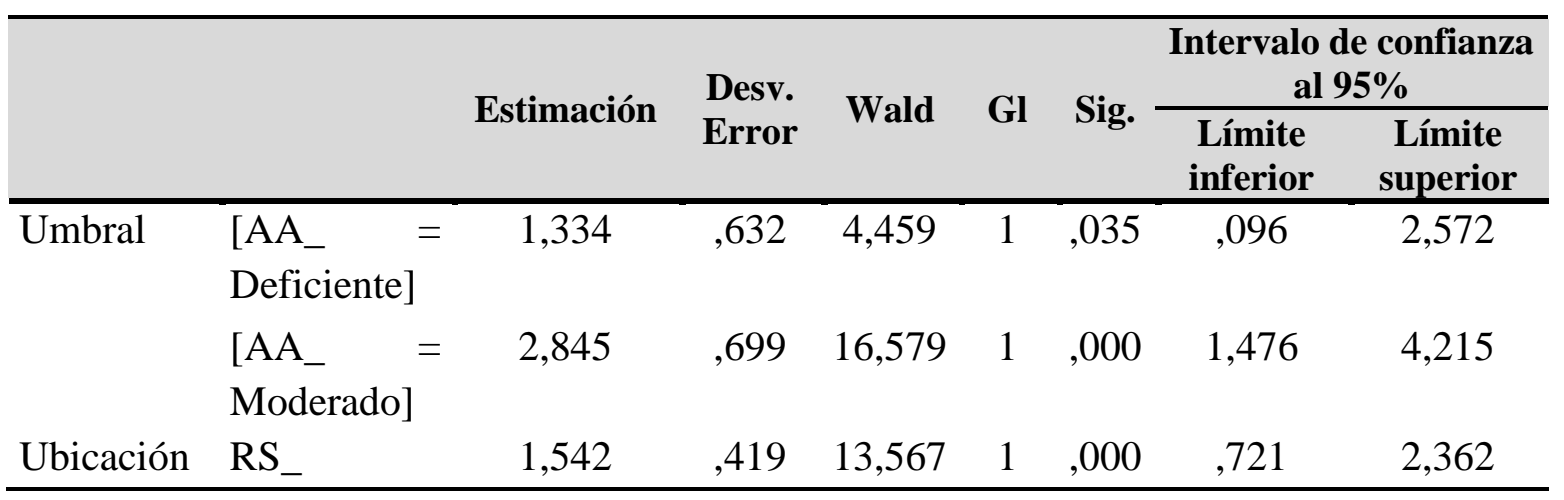

En la tabla 13, los valores de las significancias tanto para el nivel deficiente como el nivel moderado de la auditoría ambiental (variable dependiente) fueron 0,035 y 0,000 respectivamente, lo cual cumple con la condición de ser menor a 0,05; asimismo, los 
coeficientes Wald fueron 4,459 y 16,579 respectivamente, lo cual cumple con la condición de ser mayor a 4; por lo tanto, estos niveles (deficiente y moderado) fueron incididos de modo significativo por la responsabilidad social (variable independiente).

Por consiguiente, se rechazó Ho y aceptó H1.

Asimismo, en la Prueba específica 3 se tiene que:

Ho: La responsabilidad social no incide de modo significativo en el análisis del ciclo de vida. Atavillos bajo, Lima 2021.

H1: La responsabilidad social incide de modo significativo en el análisis del ciclo de vida. Atavillos bajo, Lima 2021.

\section{Tabla 14}

Determinación del ajuste de la responsabilidad social que incide en la tercera hipótesis

\begin{tabular}{lcccc}
\hline \multicolumn{1}{c}{ Modelo } & $\begin{array}{c}\text { Logaritmo de la } \\
\text { verosimilitud -2 }\end{array}$ & Chi-cuadrado & Gl & Sig. \\
\hline Sólo intersección & 32,884 & & & \\
Final & 15,445 & 17,439 & 1 &, 000 \\
\hline
\end{tabular}

De acuerdo a los resultados de la tabla 14 se observa que el valor de la significancia $0,000<0,05$ por lo tanto se rechaza la hipótesis nula y se acepta la hipótesis alterna, es decir la responsabilidad social incide de modo significativo en el análisis del ciclo de vida, Atavillos bajo, Lima 2021.

\section{Tabla 15}

Pseudo coeficiente de determinación de las variables para la tercera hipótesis

\begin{tabular}{lr}
\hline Cox y Snell &, 187 \\
Nagelkerke &, 235 \\
McFadden &, 130 \\
\hline
\end{tabular}

En la tabla 15 se observa según el valor del Pseudo R cuadrado de Nagelkerke, que la responsabilidad social incide en un $23.5 \%$ en el análisis del ciclo de vida, Atavillos bajo, Lima 2021. 


\section{Tabla 16}

Estimaciones de parámetros para la hipótesis específica 3

\begin{tabular}{llccccccc}
\hline & & $\begin{array}{c}\text { Estimac } \\
\text { ión }\end{array}$ & $\begin{array}{c}\text { Desv. } \\
\text { Error }\end{array}$ & Wald & gl & Sig. & \multicolumn{2}{c}{$\begin{array}{c}\text { Intervalo de } \\
\text { confianza al 95\% }\end{array}$} \\
\hline Umbral & $\begin{array}{l}\text { Límite } \\
\text { inferior }\end{array}$ & $\begin{array}{c}\text { Límite } \\
\text { superior }\end{array}$ \\
\hline & $\begin{array}{l}\text { Deficiente }] \\
{\left[\mathrm{ACV}_{-}=\right.}\end{array}$ & 3,191 &, 780 & 16,714 & 1 &, 000 & 1,661 & 4,720 \\
& $\begin{array}{l}{\left[\mathrm{ACV}_{-}=\right.} \\
\text {Moderado] }\end{array}$ & 6,548 & 1,075 & 37,101 & 1 &, 000 & 4,441 & 8,655 \\
\multirow{2}{*}{ Ubicación } & $\mathrm{RS}_{-}$ & 1,825 &, 461 & 15,682 & 1 &, 000 &, 922 & 2,729 \\
\hline
\end{tabular}

En la tabla 16, los valores de las significancias tanto para el nivel deficiente como el nivel moderado del análisis del ciclo de vida (variable dependiente) fueron 0,000, lo cual cumple con la condición de ser menor a 0,05; asimismo, los coeficientes Wald fueron 16,714 y 37,101 respectivamente, lo cual cumple con la condición de ser mayor a 4; por lo tanto, estos niveles (deficiente y moderado) fueron incididos de modo significativo por la responsabilidad social (variable independiente).

Por consiguiente, se rechazó Ho y aceptó H1.

Y finalmente en cuanto a la Prueba específica 4 se tiene que:

Ho: La responsabilidad social no incide de modo significativo en el sistema de gestión ambiental. Atavillos bajo, Lima 2021.

H1: La responsabilidad social incide de modo significativo en el sistema de gestión ambiental. Atavillos bajo, Lima 2021.

\section{Tabla 17}

Determinación del ajuste de la responsabilidad social que influye en la cuarta hipótesis

\begin{tabular}{lcccc}
\hline \multicolumn{1}{c}{ Modelo } & $\begin{array}{c}\text { Logaritmo de la } \\
\text { verosimilitud -2 }\end{array}$ & Chi-cuadrado & Gl & Sig. \\
\hline Sólo intersección & 21,378 & & & \\
Final & 10,748 & 10,630 & 1 &, 001 \\
\hline
\end{tabular}

De acuerdo a los resultados de la tabla 17 se observa que el valor de la significancia $0,001<0,05$ por lo tanto se rechaza la hipótesis nula y se acepta la hipótesis alterna, es decir la responsabilidad social incide de modo significativo en el sistema de gestión ambiental, Atavillos bajo, Lima 2021. 


\section{Tabla 18}

Pseudo coeficiente de determinación de las variables para la cuarta hipótesis

\begin{tabular}{lc}
\hline Cox y Snell &, 119 \\
Nagelkerke &, 270 \\
McFadden &, 219 \\
\hline
\end{tabular}

En la tabla 18 se observa según el valor del Pseudo R cuadrado de Nagelkerke, que la responsabilidad social incide en un $27.0 \%$ en el sistema de gestión ambiental, Atavillos bajo, Lima 2021.

\section{Tabla 19}

Estimaciones de parámetros para la hipótesis específica 4

\begin{tabular}{|c|c|c|c|c|c|c|c|c|}
\hline & & \multirow{2}{*}{$\begin{array}{l}\text { Estimac } \\
\text { ión }\end{array}$} & \multirow{2}{*}{$\begin{array}{l}\text { Desv. } \\
\text { Error }\end{array}$} & \multirow{2}{*}{ Wald } & \multirow{2}{*}{ gl } & \multirow{2}{*}{ Sig. } & \multicolumn{2}{|c|}{$\begin{array}{c}\text { Intervalo de } \\
\text { confianza al } 95 \%\end{array}$} \\
\hline & & & & & & & $\begin{array}{l}\text { Límite } \\
\text { inferior }\end{array}$ & $\begin{array}{c}\text { Límite } \\
\text { superior }\end{array}$ \\
\hline \multirow[t]{4}{*}{ Umbral } & {$\left[\mathrm{SGA}_{-}=\right.$} & 8,484 & 2,545 & 11,112 & 1 & ,001 & 3,496 & 13,472 \\
\hline & Deficiente] & & & & & & & \\
\hline & {$\left[\mathrm{SGA}_{-}=\right.$} & 10,864 & 3,036 & 12,802 & 1 &, 000 & 4,913 & 16,815 \\
\hline & Moderado] & & & & & & & \\
\hline Ubicación & $\mathrm{RS}_{-}$ & 3,213 & 1,220 & 6,940 & 1 & ,008 &, 823 & 5,604 \\
\hline
\end{tabular}

En la tabla 19, los valores de las significancias tanto para el nivel deficiente como el nivel moderado del sistema de gestión ambiental (variable dependiente) fueron 0,001 y 0,000 respectivamente, lo cual cumple con la condición de ser menor a 0,05; asimismo, los coeficientes Wald fueron 11,112 y 12,802 respectivamente, lo cual cumple con la condición de ser mayor a 4; por lo tanto, estos niveles (deficiente y moderado) fueron incididos de modo significativo por la responsabilidad social (variable independiente). Por consiguiente, se rechazó Ho y aceptó H1.

\section{Discusión}

A partir de los hallazgos mostrados en el capítulo precedente los niveles de responsabilidad social en los pobladores de la comunidad de Florida correspondiente a la jurisdicción de Atavillos Bajo, es catalogado mayoritariamente (50.6\%) deficiente, y lo mismo sucede con cada una de sus dimensiones, y esto se condice con los hallazgos 
de Curi (2018) quien afirma que la existencia de la responsabilidad social por parte de la entidad es intrascendente, en vista de su poca notoriedad práctica y efectiva frente a la problemática ambiental.

Además, en cuanto a la variable gestión ambiental los resultados son muy similares, es así que el $77.4 \%$ de los pobladores señala que esta en un nivel deficiente. Del mismo modo se observa una recurrencia mayoritaria del nivel deficiente especialmente en donde las dimensiones, la primera de ellas referida a la evaluación del impacto ambiental, mientras que la segunda está referida a la auditoría ambiental.

Los resultados expuestos coinciden ampliamente con los hallazgos de Marcelo (2018), no sólo en la recurrencia de los niveles sino incluso en el aspecto porcentual, tal es así que en cuanto a la responsabilidad social el $97.5 \%$ por ciento de los pobladores de San Juan de Lurigancho refieren que la responsabilidad no es de nivel alto. Asimismo, en cuanto a la gestión el $88.8 \%$ de los elementos muestrales refieren que esta no es de nivel alto.

Como se puede apreciar los resultados son muy similares, pese a tener que reconocer que los participantes de la investigación corresponden a jurisdicciones distintas e incluso en cuanto a su idiosincrasia, ya que la primera corresponde a pobladores de una comunidad rural de la provincia de Lima, sin embargo, el otro grupo corresponde a trabajadores de la municipalidad de San Juan de Lurigancho, el cual es el distrito con mayor densidad poblacional de la capital.

En tal sentido, es posible manifestar a partir de los resultados que la problemática vinculada a las variables transversal en la realidad nacional coincidiendo plenamente con lo señalado por el Organismo Mundial del Turismo (OMT) quien en 2018 afirmó que el Perú se encuentra entre los primeros lugares en el ranking de países en los más contaminados, lo cual limita la afluencia de turistas, en consecuencia, el desarrollo del país. Y considerando que la comunidad de Florida cuenta con un impresionante atractivo turístico, la problemática identificada no permitiría desarrollar la actividad turística como tal, la cual se reconoce como una alternativa que promueve el desarrollo sostenible de la comunidad, tal y como lo confirman los hallazgos de López (2018).

Además, es preciso señalar que los resultados porcentuales descritos líneas arriba colisionan con la afirmación de López y Cabrera (2021) e incluso Viorel (2019), toda vez que se considera que los hombres y mujeres como actores sociales deben respetar su 
entorno natural, cuyas acciones llegan incluso al punto de establecer una sinergia con el medio ambiente, que para el caso de la presente investigación claramente no es lo que se ha evidenciado coincidiendo con Guillen (2019) y la problemática de las organizaciones y su falta de compromiso con la conservación del medio ambiente, y más aún cuando se hace referencia que la responsabilidad social se viene aplicando no solo en el ámbito privado sino también en las instituciones públicas con una mayor importancia en los últimos años (Rakan et al., 2020).

En cuanto a los resultados inferenciales que permitieron la contrastación de la de la hipótesis general se tiene que a partir del coeficiente de determinación de Nagelkerke es posible afirmar que la responsabilidad social explica probabilísticamente en un $43.9 \%$ la variabilidad de la gestión ambiental, y considerar un ajuste adecuado del modelo (pvalor < 0.05), y según el índice de Wald, la implementación adecuada de la responsabilidad social explica probablemente en un nivel moderado de la aplicación de la gestión ambiental.

Estos resultados concuerdan con lo manifestado por Reyes (2017) quien refiere que forma parte de la política institucional la implementación en sus documentos de gestión la responsabilidad social y aún con mayor énfasis la gestión ambiental, sin embargo, la presencia de niveles de incidencia probabilística de nivel moderado se debe a la falta de vigilancia en los procesos de implementación de dichas políticas, que permitirían un manejo adecuado del cuidado medio ambiental.

En lo que respecta a los hallazgos correspondientes al primer objetivo específico el cual está referido a demostrar la incidencia de la responsabilidad social en la gestión ambiental, tenemos que, como ya se había puesto en manifiesto el nivel de la responsabilidad social se concentra en los niveles deficiente $(50.0 \%)$ y moderado $(47,6 \%)$, mientras que en cuanto a la dimensión evaluación del impacto ambiental correspondiente a la variable dependiente gestión ambiental que se realiza en la comunidad de la Florida en el 2021, se tiene que el 71.4\% de los encuestados perciben que la evaluación del impacto ambiental es de nivel deficiente, el $26.2 \%$ señalan que este es de nivel moderado, mientras que una minoría (2.4\%) afirma que la dimensión evaluación del impacto ambiental es eficiente. Estos resultados concuerdan plenamente con los hallazgos de Curi (2018) quien refiere que un porcentaje superior al $50.0 \%$ de 
los encuestado perciben que la evaluación del impacto ambiental es de tendencia negativa.

En cuanto a los resultados descritos estos coinciden con los hallazgos de Marcelo (2018) en la prevalencia del nivel bajo (58.8\%) que podría considerarse equivalente con el nivel deficiente de la presente investigación, además, en menor proporción $(30.0 \%)$ en el nivel moderado y solo un $11.3 \%$ en cuanto al nivel alto.

Estos resultados permiten colegir la existencia de un riesgo de nivel alto en el que se encuentra la comunidad y su atractivo turístico, al punto de que se podría recaer en una situación de carácter irreversible, Ya que al considerar la percepción de los elementos muestrales sobre la responsabilidad social y su incidencia en el evaluación del impacto ambiental, lo que se ha evidenciado es la existencia de un desconocimiento de la importancia de realizar una evaluación del impacto ambiental, al punto de considerar un desconocimiento o falta de aplicación de la normativa, lo que estaría afectando el medio ambiente.

En este sentido Saavedra (2021) afirma que, si es que no se aplica una adecuada metodología como la establecida en la SEIA (Sistema Nacional de Evaluación del Impacto Ambiental), o en su defecto la matriz de causa-efecto de Leopold (criterios de objetividad, método indirecto) y/o la matriz Conesa (criterios de objetividad, método directo), no es posible bajo ningún punto de vista la elaboración de planes que puedan mitigar la problemática ambiental analizada.

Así mismo, coincide con las conclusiones a las que arribó Bendezú (2020) quien refiere que para poder conseguir un medio ambiente equilibrado que ofrezca por sobre todo impactos positivos, es necesario que se desarrollen evaluaciones periódicas del impacto ambiental, y ello es responsabilidad de la gestión municipal como parte de la responsabilidad social con la comunidad a la cual representa, permitiendo de algún modo el desarrollo de sus pobladores, y como consecuencia mejorar sustancialmente los niveles de calidad de vida de la comunidad de la Florida.

En cuanto a los resultados inferenciales que permitieron la contrastación de la primera hipótesis específica se tiene que a partir del coeficiente de determinación de Nagelkerke es posible afirmar que la responsabilidad social explica probabilísticamente en un $15.1 \%$ la variabilidad de la dimensión evaluación del impacto ambiental correspondiente a la variable gestión ambiental, y considerar un ajuste adecuado del 
modelo (p-valor < 0.05), y según el índice de Wald, la implementación adecuada de la responsabilidad social explica probablemente en un nivel moderado de la implementación de la evaluación del impacto ambiental. Se halla similitud con lo que afirma Curi (2018) al referirse que la evaluación del impacto ambiental no viene teniendo una aplicación apropiada, por lo que su incidencia es mínima en el mejoramiento del medio ambiente.

En lo que respecta a los hallazgos correspondientes al segundo objetivo específico el cual está referido a demostrar la incidencia de la responsabilidad social en la auditoría ambiental, tenemos que, como ya se había evidenciado el nivel de la responsabilidad social se concentra en los niveles deficiente $(50.0 \%)$ y moderado $(47,6 \%)$, mientras que en cuanto a la dimensión auditoría ambiental correspondiente a la variable dependiente gestión ambiental que se realiza en la comunidad de la Florida (jurisdicción del distrito de Atavillos Bajo) en el 2021, se tiene que el 29.8\% de los encuestados perciben que la auditoría ambiental es de nivel deficiente, mientras que el $31.0 \%$ señalan que es de nivel moderado, mientras que mayoritariamente $(39.3 \%)$ los pobladores encuestados afirman que la dimensión auditoría ambiental es eficiente.

Los resultados expuestos en el párrafo precedente son contrarios a los mostrado por Curi (2018) quien señala que los encuestados perciben que las técnicas y procedimientos que se han utilizado para la ejecución de la autoría ambiental no han resultados ser las más adecuadas (59.4\%), esta diferencia sustancial en los hallazgos es posible esbozar una explicación certera, ya que en el primer caso, pobladores de la comunidad de Florida, no están involucrados en la gestión municipal, por lo que sus percepciones varían respecto de aquellos que han sido encuestados y resultaron ser trabajadores de la entidad, en tal razón al formar parte de la misma organización les ha permitido una apreciación más objetiva. En donde se verifica cierta coincidencia es en afirmar que resulta importante el empleo de la auditoría ambiental en las instituciones, ya que todas en su rango de acción repercuten en el medio ambiente.

En cuanto a los resultados inferenciales que permitieron la contrastación de la segunda hipótesis específica se tiene que a partir del coeficiente de determinación de Nagelkerke es posible afirmar que la responsabilidad social explica probabilísticamente en un 18.9\% la variabilidad de la dimensión auditoría ambiental correspondiente a la variable gestión ambiental, y considerar un ajuste adecuado del modelo (p-valor $<0.05$ ), y según 
el índice de Wald, la implementación adecuada de la responsabilidad social explica probablemente en un nivel moderado de la auditoría ambiental.

En este sentido, la casi inexistente implementación de la responsabilidad social en la entidad municipal advierte la ausencia de auditorías ambientales (Curi, 2018), por lo que esta situación impide que se cuente con evidencias del nivel de impacto (positivos o negativos) en el medio ambiente de la comunidad de la Florida. Cabe precisar que los resultados descritos contravienen una de las conclusiones de Aspajo (2017) quien en su investigación referida a la auditoría ambiental señala que este si influye significativamente ( $\mathrm{p}$-valor $<0.05$ ) en la responsabilidad social.

Esta última afirmación puede ser explicada como contraria a los hallazgos del presente estudio, toda vez que el estudio que desarrollo el citado investigador está referido al contexto minero, y como se sabe en este escenario la auditoría ambiental resulta clave, por la regulación existente en el sector, sino también para con su relación con la comunidad la cual se haya en el rango del impacto de las actividades extractivas. En esta misma línea los investigadores Palencia et al. (2018) señalan que la auditoría ambiental es parte importante para el desarrollo ya que permite una relación armoniosa entre el ambienta, la sociedad y la empresa, logrando coincidir con lo referido por Cabrera (2019).

En lo que respecta a los hallazgos correspondientes al tercer objetivo específico el cual está referido a demostrar la incidencia de la responsabilidad social en el análisis del ciclo de vida, tenemos que, como ya se había evidenciado el nivel de la responsabilidad social se concentra en los niveles deficiente $(50.0 \%)$ y moderado $(47,6 \%)$, mientras que en cuanto a la dimensión análisis del ciclo de vida correspondiente a la variable dependiente gestión ambiental que se realiza en la comunidad de la Florida (jurisdicción del distrito de Atavillos Bajo) en el 2021, se tiene que el 58.3\% de los encuestados perciben que el análisis del ciclo de vida es de nivel deficiente, mientras que el $38.1 \%$ señalan que es de nivel moderado, mientras que una porción mínima (3.6\%) de los pobladores encuestados afirman que la dimensión análisis del ciclo de vida es eficiente. En vista que la comunidad en donde se desarrolló el estudio está vinculado a la actividad turística ya que cuenta con un atractivo turístico importante y su limitado desarrollo pese a la gran potencialidad con el que cuenta, es así que al hacer referencia al análisis del ciclo de vida se está enfatizando en el producto turístico. En tal sentido, 
los resultados descriptivos del párrafo precedente no hacen más poner de manifiesto el casi inexistente análisis, que tiene que ver con las etapas de desarrollo, introducción, crecimiento, madurez y declive de cualquier producto incluso el turístico.

Sin embargo, si bien ningún producto es para siempre, López (2018) señala que la actividad turística es una de las pocas actividades económicas sostenibles, de las cuales ya algunos países han logrado desarrollarse (Croacia Tailandia, Jamaica e Islandia. Sin embargo, los resultados muestran que, para la gestión municipal, a menos desde la percepción de los pobladores de la comunidad que fueron encuestados, esta actividad no se encuentra entre sus prioridades, ya que más de la mitad de los encuestados señalan que este análisis es deficiente, y se hace notorio ya que el atractivo turístico se encuentra casi en el anonimato, como es el caso de: Ruinas de Rupac, conocido también como el Machu Picchu limeño.

En cuanto a los resultados inferenciales que permitieron la contrastación de la tercera hipótesis específica se tiene que a partir del coeficiente de determinación de Nagelkerke es posible afirmar que la responsabilidad social explica probabilísticamente en un 23.5\% la variabilidad de la dimensión análisis del ciclo de vida correspondiente a la variable gestión ambiental, y considerar un ajuste adecuado del modelo ( $\mathrm{p}$-valor $<0.05$ ), y según el índice de Wald, la implementación adecuada de la responsabilidad social explica probablemente en un nivel moderado de la implementación del análisis del ciclo de vida. De los resultados inferenciales se puede colegir también que una deficiente responsabilidad ambiental genera probablemente un inadecuado análisis del ciclo de vida del producto más importante de la comunidad, como es el turismo.

Los hallazgos concuerdan ampliamente con las afirmaciones de Haro (2018) quien señala que para que existe una adecuada gestión ambiental resulta necesario el desarrollo de la conciencia ambiental, lo cual implica que cuando este aspecto es desatendido, es decir no se promueve la concientización en la comunidad, la contribución de esta en cada una de las etapas que analiza el ciclo de vida es casi nula, finalmente afirma que esta inacción está asociada a la comportamiento de la entidad municipal y/o de la gerencia responsable de la temática ambiental, coincidiendo con la afirmación de Pinzón (2017).

En lo que respecta a los hallazgos correspondientes al cuarto objetivo específico el cual está referido a demostrar la incidencia de la responsabilidad social en el sistema de 
gestión ambiental, tenemos que, como ya se había evidenciado el nivel de la responsabilidad social se concentra en los niveles deficiente $(50.0 \%)$ y moderado (47,6\%), mientras que en cuanto a la dimensión sistema de gestión ambiental correspondiente a la variable dependiente gestión ambiental que se realiza en la comunidad de la Florida (jurisdicción del distrito de Atavillos Bajo) en el 2021, se tiene que el $92.9 \%$ de los encuestados perciben que el sistema de gestión ambiental es de nivel deficiente, mientras que el $6.0 \%$ señalan que es de nivel moderado, mientras que una porción mínima (1.2\%) de los pobladores encuestados afirman que la dimensión sistema de gestión ambiental es eficiente.

Los resultados expuestos son contrarios a los hallazgos de Aspajo (2017) quien en su investigación refiere que el $80.7 \%$ de los encuestados afirman que el sistema de gestión ambiental es efectivo. Esta afirmación se explica ya que el escenario de estudio difiere entre las investigaciones comparadas, en el caso de Aspajo hace referencia al contexto minero, muy distinta la locación en la que se ha desarrollado el presente estudio, y más aún en vista de lo sensible que es la problemática ambiental en el contexto minero, en tal sentido los sistemas de gestión ambiental se esperan que sean efectivos, tal como es el caso comparado. Además, Saldivar (2019) enfatiza en la importancia de la implementación de un sistema de gestión ambiental ya que contribuye con el desarrollo económico, social y ambiental, por lo que se puede colegir de los hallazgos donde los encuestados califican de deficiente la implementación de dicho sistema, concuerda con la repercusión en el letargo en el que se encuentra la comunidad rural de la Florida.

En cuanto a los resultados inferenciales que permitieron la contrastación de la cuarta hipótesis específica se tiene que a partir del coeficiente de determinación de Nagelkerke es posible afirmar que la responsabilidad social explica probabilísticamente en un $27.0 \%$ la variabilidad de la dimensión sistema de gestión ambiental, y considerar un ajuste adecuado del modelo (p-valor < 0.05), y según el índice de Wald, la implementación adecuada de la responsabilidad social explica probablemente en un nivel moderado del sistema de gestión ambiental. Mientras que, los resultados inferenciales descritos por Aspajo (2017) son aún más contundentes, ya que afirma que la efectividad de la implementación de un sistema de gestión ambiental a partir del coeficiente de Contingencia C (0.56; asociación alta) concluye en que existe una influencia positiva en el desarrollo sostenible. 


\section{CONCLUSIÓN O CONSIDERACIONES FINALES}

\section{Primera}

La responsabilidad social incide significativamente de modo probabilístico con un 43.9\% en la gestión ambiental en el distrito de Atavillos en el año 2021; con lo cual la relación causal de la hipótesis general queda demostrada (Wald: 17,324; gl: 1 y p: ,000).

\section{Segunda}

La responsabilidad social se incide significativamente con un $15.1 \%$, en la dimensión evaluación del impacto ambiental de la gestión ambiental en el distrito de Atavillos en el año 2021; con lo cual la relación causal de la primera hipótesis especifica queda demostrada (Wald: 29,237; gl: 1 y p: ,000).

\section{Tercera}

La responsabilidad social incide significativamente de modo probabilístico con un $18.9 \%$, en la dimensión auditoría ambiental de la gestión ambiental en el distrito de Atavillos en el año 2021; con lo cual la relación causal de la segunda hipótesis especifica queda demostrada (Wald: 16,579; gl: 1 y p: ,000).

\section{Cuarta}

La responsabilidad social incide significativamente de modo probabilístico con un $23.5 \%$, en la dimensión análisis del ciclo de vida de la gestión ambiental en el distrito de Atavillos en el año 2021; con lo cual la relación causal de la tercera hipótesis especifica queda demostrada (Wald: 37,101; gl: 1 y p: ,000).

\section{Quinta}

La responsabilidad social incide significativamente de modo probabilístico con un $27.0 \%$ en la dimensión sistema de gestión ambiental de la gestión ambiental en el distrito de Atavillos en el año 2021; con lo cual la relación causal de la cuarta hipótesis especifica queda demostrada (Wald: 12,802; gl: 1 y p: ,001).

\section{LISTA DE REFERENCIAS}

Ansong, A., \& Nisar, T. (2017). Corporate social responsibility and access to finance among Ghanaian SMEs: The role of stakeholder engagement. Cogent Business \& Management, 4(1), 1-N.PAG. Shorturl.at/dtzEY

Álvarez, I. (2017). Gobernanza Urbana, responsabilidad social empresarial y participación ciudadana: Creo Antofagasta en la disputa por hegemonía de la 
gran minería [Tesis de pregrado, Universidad de Chile Santiago de Chile]. Repositorio institucional. Shorturl.at/zBRWX

Abreu, J. L. (2012). La Formulación de los Antecedentes del Problema de Investigación Científica. Revista Daena (International Journal of Good Conscience), 7(1), 163-168. Shorturl.at/ajmuF

Ahn, J., Shamin, A., Park, J. (2020). Impacts of cruise industry corporate social responsibility reputation on customer's loyalty. International Journal of hotel management. 92(1),1. Shorturl.at/pHQV7

Ajina, A., Roy, S., Nguyen, B., Japutra, A. (2020). Brand value enhancements through corporate social responsibility initiatives. Qualitative Market Research: An International Journal. 1(1), 1352- 2752. Shorturl.at/mJW34

Amaiquema Marquez, F. A., Vera Zapata, J. A., \& Zumba Vera, I. Y. (2019). Enfoques para la formulación de la hipótesis en la investigación científica. Revista Conrado, 15(70), 354-360. Shorturl.at/adzI8

Pedersen, E. R. G., Gwozdz, W., \& Hvass, K. K. (2018). Exploring the Relationship Between Business Model Innovation, Corporate Sustainability, and Organisational Values within the Fashion Industry. Journal of Business Ethics, 149(2), 267-284. Shorturl.at/etCK3

Aspajo, N. T., (2017). La auditoría ambiental y su influencia en la responsabilidad social empresarial del sector minero. Perú 2010 - 2016 [Tesis Doctoral, Universidad San Martín de Porres]. Repositorio institucional SMP. Shorturl.at/rNW16.

Babu, N., Roeck, k., Raineri, N. (2020). Implications for the social responsibility of employees. Journal of business research. Shorturl.at/jqGHV

Banchón, H. A.; Nauta, V. R, (2018). Auditoría ambiental para el cumplimiento del plan de manejo ambiental, de la planta de beneficio Eninsatch S.A. [Tesis Magister, Universidad de las Fuerzas Armadas, Ecuador]. Repositorio institucional UFA. Shorturl.at/lsQ17

Bendezú, C. A., (2020). Evaluación del impacto ambiental por la extracción de canteras de arena del centro poblado Peña Negra, San Juan Bautista, 2020 [Tesis Magister, Universidad Científica del Sur]. Repositorio institucional UCS. Shorturl.at/rBDOX 
Benítez, J., Ruiz, L., Castillo, A., Llorens, J. (2020). How Corporate Social Responsibility Activities Influence Employer Reputation: The Role of Social Media Capability. Journal of Cleaner Production. Shorturl.at/tCIP5

Cabrera, J. E., (2019). La gestión de riesgo de desastres y la responsabilidad social en el gobierno regional de Tacna [Tesis de maestría, Universidad Nacional Jorge Basadre Grohmann]. Shorturl.at/boKRT

Carbajal, A. B., (2018). Análisis de ciclo de vida de las fases de aprovechamiento y reciclaje del sistema de gestión integral de residuos sólidos municipales del Cantón Mejía [Tesis Magister, Repositorio Universidad de las Fuerzas ArmadasEcuador]. Repositorio Institucional UFA. Shorturl.at/quNR6

Carmona Caldera, C. (2020). Evaluación ambiental, Consulta indígena Y EL "Desplazamiento" de los derechos de los pueblos Indígenas. (Concepción), 88(248), 199-232. Shorturl.at/bemL9

Carlos, M. J., (2018). Propuesta de un Modelo de Gestión Ambiental Institucional, basado en la Teoría Ecológica de los Sistemas de Bronfenbrenner, para mejorar los hábitos de conservación del medio ambiente en niños del nivel inicial [Tesis Doctoral, Universidad Cesar Vallejo]. Repositorio institucional UCV. Shorturl.at/nsDGT

Cayo, W. (2018). Gestión por resultados y administración pública en la Policía Nacional del Perú, Lima - 2018 [Tesis Doctoral, Universidad Cesar Vallejo]. Repositorio institucional UCV Cesar Vallejo. Shorturl.at/fpAIZ

Castro-Gonzáles, S., Bande, B., Fernández-Ferrín, P., Kimura, T. (2019). Behaviors of corporate social responsibility and consumer protection: the importance of emotions and moral virtues. Journal of Cleaner Production. 231(1), 846-855. Shorturl.at/oBCOZ

Caruana, A., Vella, J., Konietzn, J., Chircop, S. Corporate greed: its effect on customer satisfaction, corporate social responsibility and corporate reputation among bank customers. Springer. Journal of Financial Services Marketing. Shorturl.at/almEW

Cesar, S., Jhony, O. (2020). Corporate social responsibility supports the construction of strong social capital in the mining context. Journal of Cleaner Production. 267(1), 122-162. Shorturl.at/qwxJZ 
Contini, M., Annunziata, E., Rizzi, F., Frey, M. (2020). Exploring the influence of corporate social responsibility domains on consumer loyalty. Journal of Cleaner Production. Shorturl.at/gxGL7

Cueto, C. (2017). La responsabilidad social corporativa del sector público: un análisis aplicado a las grandes ciudades en España [Tesis Doctoral, Universidad de Zaragoza]. Repositorio institucional UZME. Shorturl.at/bjnNZ

Curi, D. M., (2018). La auditoría ambiental y la gestión de las empresas del sector minero de las regiones Junín y Cajamarca 2016-2017 [Tesis Doctoral, Universidad San Martín de Porres]. Repositorio institucional UMSP. Shorturl.at/kxBJY

Chibás-Creagh, Mario, \& Gómez-Martínez, Felicia del Carmen (2019). La sostenibilidad del Medio Ambiente. Una vía desde la enseñanza de la Botánica. Universidad de Holguín Oscar Lucero Moya. Shorturl.at/rABN2

Dextre, R. M., (2020). Análisis de ciclo de vida (ACV) del manejo de residuos de aparatos eléctricos y electrónicos (RAEE) por la empresa operadora de residuos Comimtel S.A.C., Lima, periodo 2017-2019 [Tesis Pregrado, Universidad Nacional Antúnez de Mayolo]. Repositorio institucional UNAM, DOI

Diem, L. (2016). Customer relationship Enhancements from corporate social. Business Source Premier. 10.1057/s41299-016-0001-4.

Ferreyro, A., \& Longhi, A. L. D. (2014). Metodología de la investigación. Encuentro Grupo Editor.responsibility activities whitim the hospital sector. Palgrave. Encuentro Grupo Editor, 2014. ISBN 9789871432660. Shorturl.at/uILMU

Ferrell, O. C., Harrison, D. E., Ferrell, L., \& Hair, J. F. (2019). Business ethics, corporate social responsibility, and brand attitudes: An exploratory study. Journal of Business Research, 95, 491-501.DOI10.1016/j.jbusres.2018.07.039

Gadea, F. G., Cuenca, R. C., y Montero, A. (2019). Epistemología y Fundamentos de la Investigación Científica. Cengage Learning Editores, S.A. de C.V. http://latinoamerica.cengage.com

Granada, L., Álvarez, N., Afanador, M. (2018). Lineamientos para la implementación de una filosofía de gestión ambiental: Ediciones de la $U$. 
González-Abreu, Betty, \& Ochoa-Ávila, Migdely Barbarita, \& Quesada-Musa, Gizne de las Mercedes (2019). La gestión ambiental integral en el hotel Brisas Covarrubias de Las Tunas. Ciencias Holguín, 25(1),69-83. ISSN. https://www.redalyc.org/articulo.oa?id=181558076007

Gomez Luna, Liliana María. (2020). El desafío ambiental: enseñanzas a partir de la COVID-19. MEDISAN, 24(4), 728-743. Epub 24 de julio de 2020. Shorturl.at/nsR08

Guillen, M. A., (2019). Proyectos de inversión con responsabilidad social empresarial y su incidencia en los grupos de interés (stakeolders) de las grandes industrias del sector alimentos del Ecuador [Tesis doctoral, Universidad Nacional Mayor de San Marcos]. Repositorio Institutional UNMSM. https://unmsm.edu.pe/handle/20.500.12672/11196

Gul, F., Shams, S. (2020). Corporate Social Responsibility, Overconfident CEOs, and Empire Building. Journal of Business Research 111 1(1), 52-68. Shorturl.at/Zfmny

Muflih, M. (2021). The link between corporate social responsibility and customer loyalty: Empirical evidence from the Islamic banking industry. Journal of Retailing \& Consumer Services, 61, N.PAG. Shorturl.at/ftwH0

Hadj, B. (2020). Effects of corporate social responsibility towards stakeholders and environmental management on responsible innovation and competitiveness. Journal of Cleaner Production. 1(1), 1. 34 .10.1016/j.jclepro.2019.119490

Han, S.-L., \& Lee, J. W. (2021). Does corporate social responsibility matter even in the B2B market?: Effect of B2B CSR on customer trust. Industrial Marketing Management, 93, 115-123. DOI: shorturl.at/bquCM

Haro, U. E., (2018). Gestión pública ambiental y la conciencia ambiental según servicios educativos el agustino, anexo de monterrey $i$, distrito de ate vitarte, lima, 2017 [Tesis Magister, Universidad Nacional Agraria de la Selva, Tingo María]. Repositorio UNAS. Shorturl.at/xEHMV

Hernández-Perlines, F. (2017). Influencia de la responsabilidad social en el desempeño de las empresas familiares. GCG: Revista de Globalización, Competitividad \& Gobernabilidad, 11(3), 58-73. Shorturl.at/opPUZ

Hernández, S. (2014). Metodología de la Investigación. 6ta Edición MrGraw-Hill. LAP. 
Lavado, L. (2020) Epistemología e investigación. Lima. Fondo editorial.

Hincapié M., J. P. (2017). Elementos ontológicos, epistemológicos y metodológicos para la construcción de un marco teórico de estudio de los activos intangibles. Cuadernos de Contabilidad, 18(45), 86-109. Shorturl.at/uEGO6

Hurtado, j. (2015). El proyecto de investigación. Comprensión holística de la metodología y la investigación Venezuela, Ediciones Quirón.

Jeon, M., Lee, S., Jeong, M. (2019). Perceived corporate social responsibility and customers' behaviors in the ridesharing service industry. International Journal of Hospitality Management. 84 (1) 102-34. 10.1016/j.ijhm.2019.102341

Krauskopf, E. (2018). An analysis of discontinued journals by Scopus. Scientometrics, 116(3), 1805-1815. https://doi.org/10.1007/s11192-018-2808-5

Latif, K. F., Pérez, A., \& Sahibzada, U. F. (2020). Corporate social responsibility (CSR) and customer loyalty in the hotel industry: A cross-country study. International Journal of Hospitality Management, 89, N.PAG. Shorturl.at/ctLVX

Leiva C, Frans, A. (2020). Educación Ambiental para el poblador del distrito de Casa Grande en el manejo de residuos sólidos urbanos entre julio a diciembre del año 2019. Arnaldoa, 27(1), 323-334. Shorturl.at/sEFKP

López, D. L., (2018). La actividad turística como alternativa de desarrollo local sostenible en el distrito de San Jerónimo Andahuaylas -2017, [Tesis Magister, Universidad José Carlos Mariátegui Moquegua]. Repositorio institucional JCM. Shorturl.at/iDGTV

Maldonado, J. (2018). Metodología de la Investigación Social. Paradigmas: cuantitativo, sociocrítico, cualitativo, complementario. ( $1^{\mathrm{a}}$ edición). Bogotá: Ediciones de la U.

Manzano, M. J., (2017). Evaluación del impacto de sistemas gestión ambiental en instituciones de educación superior certificados con ISO 14001. [Tesis Posgrado, Universidad de Barcelona]. Repositorio institucional. Shorturl.at/nxyQZ

Marcelo, Y. Y. (2018). Responsabilidad social en la gestión ambiental de la Municipalidad de San Juan de Lurigancho, 2018 [Tesis de maestría, Universidad Cesar Vallejo]. https://repositorio.ucv.edu.pe/handle/20.500.12692/34670 
Marques de Sousa, M., André Gouveia, B. de L., da Costa Farias Almeida, T., Moreira Freire, M. E., de Sousa, F. S., \& Oliveira, S. H. dos S. (2021). Instrumento para medir a intenção de reduzir o consumo de sal em pessoas com insuficiência cardíaca. Avances En Enfermeria, 39(3), 332-344. Shorturl.at/wyFJL

Montes, C. (2020) Generación y manejo de residuos durante la pandemia del COVID19”. Shorturl.at/szEHW

Muñoz, C. \& Pérez, S., (2020). La Responsabilidad Social en CEMEX. Investigación Valdizana, 14(4),]. ISSN: 1994-1420. Shorturl.at/iCEH6

Naciones Unidas (2020) "El cambio climático es más mortal que el Coronavirus". https://news.un.org/es/story/2020/03/1470901

Ñaupas, P. H. (2014). Metodología de la investigación científica. Lima: Universidad Nacional Mayor de San Marcos. Shorturl.at/msvCQ

Orozco-Alvarado, J., \& Díaz-Pérez, A. (2018). ¿Cómo redactar los antecedentes en una investigación cualitativa? Revista Electrónica de Conocimientos, Saberes y Prácticas, 1(2), 66-82. Shorturl.at/muKPU

Pacori, K. y Pacori, W. (2019). Metodología y diseño de la investigación científica, Eco Edit ffecaat.

Palencia, M. L., \& Ben, V. P. (2013). Ética en la investigación psicológica: una mirada a los códigos de ética de Argentina, Brasil y Colombia. Revista de Psicología (1669-2438), 9(17), 53-65. Shorturl.at/cpN04

Palencia, R. J., Huertas, S. M. y Brito, L. D. (2018). Importancia de la implementación de la auditoría ambiental en la perspectiva de la gestión ambiental de las empresas [Tesis de maestría, Universidad Cooperativa de Colombia]. Rpositorio $\mathrm{UC}$.

https://repository.ucc.edu.co/bitstream/20.500.12494/7632/1/2018_importancia_ implementacion_auditoria.pdf

Pinzón, A. Y. (2017). La responsabilidad social universitaria y la gestión ambiental en Bogotá: Estudio comparativo entre la Universidad del Rosario y la Universidad distrital Francisco José de Caldas [tesis de, Universidad La Gran Colombia]. https://repository.ugc.edu.co/bitstream/handle/11396/3875/Respossabilidad_amb iental_universitaria.pdf?sequence=1\&isAllowed=y 
Porras, E. (2016). Análisis de la aplicación de esquemas de responsabilidad social corporativa en cinco empresas de la ciudad de Quito-Ecuador como mecanismo de fortalecimiento empresarial [Tesis magister, Pontificia Universidad Católica del Ecuador]. Repositorio Institucional PUC. Shorturl.at/uxzS7

Quiroga, J. C., (2018). Mejora del proceso de recolección de residuos de manejo especial a través de la metodología DMAIC [Tesis Magister, Universidad Autónoma del estado de México, Toluca]. Repositorio Institucional UAMT

Reyes, S. (2019). Gestión eco-ambiental organizacional para el manejo efectivo del recurso agua en la empresa procesadora pesquera del Ecuador. [Tesis Doctoral, Universidad Nacional Mayor de San Marcos]. Repositorio institucional UNMSM. Shorturl.at/iADNO

Reyes, L (2017). Responsabilidad ambiental en la empresa del sector público; caso dirección de impuestos y aduanas nacionales (DIAN) Santa Marta en perspectiva de los saberes del manejo de residuos sólidos [Tesis magister, Universidad Manizales]. Repositorio UM. https://bit.ly/2YVYxO3

Rivera, J. E., (2018). Implementación del sistema de gestión ambiental ISO 14001:2015 para minimizar los impactos ambientales de la mina San Roque FM S.A.C. año 2017 [Tesis Pregrado, Universidad Nacional Santiago Antúnez de Mayolo]. Repositorio institucional UNSAM. Shorturl.at/pyU16

Rojas Vallejo, M. (2020) Covid-19 y cambio climático: crisis interdependientes. Shorturl.at/mqEMX

Rojo, N., Gallastegui, G., Encinas, M., Gómez de Balugera, Z. (2018). Gestión y evaluación de impacto ambiental: Universidad del País Vasco Euskal Herriko Unibertsitateko Argitalpen Zerbitzua. Shorturl.at/qFWX3

Oscar. (2021). Sistema de gestión de residuos sólidos. Periodo 2015-2019. Población y Desarrollo, 27(52), 15-29. [Tesis doctoral, Universidad Nacional de Asunción]. Repositorio Institucional UNA. Shorturl.at/aivMY

Saavedra, G. E., (2021). Evaluación del impacto ambiental del ecosistema frágil costero humedal Santa Julia [Tesis doctoral, Universidad Nacional de Piura]. Repositorio Institucional UNP. https://repositorio.unp.edu.pe/handle/20.500.12676/2877 
Salas-Canales, Hugo Jesús. (2021). Educación ambiental y su contribución al cuidado y protección del ecosistema. Fides et Ratio - Revista de Difusión cultural y científica de la Universidad La Salle en Bolivia,21(21), 229-246. Shorturl.at/yzFQY

Saldívar, L. R., Villar, L. y Barrios, O. (2020). Sistema de gestión de residuos. Acción por el clima. 10.18004/pdfce/2076-054x/2021.027.52.015

Soto, R. (2018). Los fundamentos de la responsabilidad social en la estrategia de la organización. Retrieved. Shorturl.at/aAKS7

Taquia, M. A., (2020).-Gestión del riesgo de desastres y la conciencia ambiental en la responsabilidad social del personal del Minagri, Lima 2020-[Tesis Doctoral, Universidad Cesar Vallejo]. Repositorio institucional UCV. Shorturl.at/qFT07

Urzaiz, L. (2016). La Responsabilidad Social Empresarial en la Prensa del Siglo XXI [Tesis Doctoral, Universidad de Sevilla]. Repositorio institucional US. Shorturl.at/jELXY

Vico, M., J. (2018). La justificación científica y filosófica del respeto hacia la naturaleza: Teilhard de Chardin, Arne Naess y el Papa Francisco. Contrastes: Revista Internacional de Filosofia, 23(1), 93-110. Shorturl.at/kBNR6

Viñan, J. (2017). Aplicación de los indicadores Eethos para fortalecer la gestión de responsabilidad social en las cooperativas de ahorro y crédito del Ecuador [Tesis Doctoral, Universidad Nacional Mayor de San Marcos]. Repositorio institucional UNMSM. shorturl.at/vwL78

Zeballos, J. \& Rentería, M. (2016) Propuesta de mejora para la gestión estratégica del programa de segregación en la fuente y recolección selectiva de residuos sólidos domiciliarios en el distrito de los Olivos [Tesis Doctoral, Pontificia Universidad Católica del Perú]. Repositorio institucional PUC. Shorturl.at/dzCF9 